Association for Information Systems AIS Electronic Library (AISeL)

Wirtschaftsinformatik Proceedings 2001

Wirtschaftsinformatik

September 2001

\title{
Individuelle Risikohandhabung bei der Zahlungssystemwahl
}

Martin Reichenbach

Universität Freiburg, marei@iig.uni-freiburg.de

Follow this and additional works at: http://aisel.aisnet.org/wi2001

\section{Recommended Citation}

Reichenbach, Martin, "Individuelle Risikohandhabung bei der Zahlungssystemwahl" (2001). Wirtschaftsinformatik Proceedings 2001. 36.

http://aisel.aisnet.org/wi2001/36

This material is brought to you by the Wirtschaftsinformatik at AIS Electronic Library (AISeL). It has been accepted for inclusion in Wirtschaftsinformatik Proceedings 2001 by an authorized administrator of AIS Electronic Library (AISeL). For more information, please contact elibrary@aisnet.org. 
In: Buhl, Hans Ulrich, u.a. (Hg.) 2001. Information Age Economy; 5. Internationale Tagung Wirtschaftsinformatik 2001. Heidelberg: Physica-Verlag

ISBN: 3-7908-1427-X

(C) Physica-Verlag Heidelberg 2001 


\title{
Individuelle Risikohandhabung bei der Zahlungssystemwahl
}

\author{
Martin Reichenbach \\ Universität Freiburg
}

\begin{abstract}
Zusammenfassung: Die Informations- und Kommunikationstechnik wird in den kommenden Jahren in der Lage sein müssen, Finanztransaktionen in weit größßerem Ausmaße abwickeln zu können, als das bisher der Fall ist. Sichere und effiziente elektronische Geld- und Zahlungssysteme sind dazu eine wesentliche Voraussetzung. Diese elektronischen Zahlungssysteme müssen Wertübertragungen zu geringen Transaktionskosten ermöglichen und sollten dabei mindestens vergleichbare Sicherheitsniveaus wie die herkömmlichen Zahlungsinstrumente Bargeld, Rechnung oder die kontobasierte Überweisung bieten. Im Hinblick auf die Vielzahl vorhandener elektronischer Zahlungssysteme und speziell der möglichen Folgen ihrer Verwendung herrschen bei den Endkunden bzw. Nutzern jedoch Intransparenz und Verunsicherung.

Ausgehend von dieser Problemstellung werden in diesem Beitrag die Voraussetzungen für eine nutzerorientierte individuelle Risikohandhabung bei der Auswahl elektronischer Zahlungssysteme skizziert.
\end{abstract}

Schlüsselworte: Elektronische Zahlungssysteme, Mehrseitige Sicherheit, Risikomanagement, Entscheidungsunterstützung, Bewertung

\section{Einleitung}

Die Potentiale der Informations- und Kommunikationstechnik für elektronisches Bezahlen werden bisher noch nicht ausgeschöpft. So zeigt sich für die Zahlungsabwicklung ein Beharrungsvermögen zugunsten traditioneller Methoden, wie etwa das Bezahlen per Vorkasse, per Nachnahme oder per Rechnung. Die für das Internet entwickelten elektronischen Zahlungssysteme werden dagegen kaum verwendet.

Als mögliche Ursache für diese Stagnation ist in empirischen Untersuchungen herausgefunden worden, dass den Endkunden bzw. Nutzern immer noch das Vertrauen in die korrekte Funktionsweise und Ausführung der zur Zahlungsdurchführung eingesetzten Soft- und Hardware fehlt [EgEn00; EgMü01]. In weiteren Untersuchungen wird gezeigt, dass die Nutzer bei der Durchführung von Zahlungstransaktionen zunehmend die Kontrolle über ihre Privatsphäre nachfragen [Fo00]. 
Da Datensammlungen mit Nutzerinformationen für Unternehmen im Internet zusehends zu wertvollen Aktivposten werden, sind die Privatsphäre und die Kommunikationssicherheit der Nutzer in verstärktem Maße bedroht [JeMa00].

Aufgrund komplexer Informationsströme und Vorgänge beim Einsatz elektronischer Zahlungssysteme, die zwischen den verschiedenen Beteiligten ablaufen, ist Sicherheit nicht automatisch gewährleistet. So kann selbst bei den fortschrittlichsten elektronischen Zahlungssystemen die Anonymität, die Unbeobachtbarkeit und Unverkettbarkeit beim Bezahlvorgang mit Bargeld noch immer nicht in befriedigendem Maße nachgebildet werden. Im Hinblick auf die Vielzahl vorhandener elektronischer Zahlungssysteme und speziell der möglichen Folgen ihrer Verwendung herrschen bei den Nutzern Intransparenz und Verunsicherung. Kennzeichnend für diese Verunsicherung ist das Vorgehen der Nutzer beim Bezahlen im Internet: Häufig müssen sie aus den ihnen zur Verfügung stehenden Zahlungssystemen ein vorgegebenes auswählen, ohne sich dabei über die Auswirkungen dieser Auswahl auf ihre Sicherheits-, Funktionalitäts- und Wirtschaftlichkeitsanforderungen im klaren zu sein. Letztlich müssen sich die Nutzer zur Abwicklung einer Transaktion im gegenwärtigen Zustand immer „blind“ zugunsten eines der Zahlungssysteme entscheiden, die ihnen zur Verfügung stehen.

Ausgehend von dieser Problemstellung werden in diesem Beitrag die Voraussetzungen für eine nutzerorientierte individuelle Risikohandhabung elektronischer Zahlungssysteme skizziert. Der vorgestellte Ansatz unterstützt die Nutzer bei der Wahl eines Zahlungssystems, sowohl langfristig beim Aufbau des individuellen Zahlungssystemportfolios als auch kurzfristig während der Durchführung einer konkreten Zahlungstransaktion.

\section{Risiko, Risikoanalyse und Risikohandhabung}

\subsection{Der verwendete Risikobegriff}

Die hier verwendete zielorientierte Risikodefinition sieht Risiko als Gefahr einer negativen Ziel- bzw. Erwartungsabweichung. Dies ermöglicht anders als etwa die verteilungsorientierten Definitionsansätze die Berücksichtigung auch qualitativer Aspekte, etwa der informationellen Selbstbestimmung. Darunter ist auch das Recht zu fassen, Informationen nur „datensparsam“ und der Transaktion angemessen abzugeben.

Als Ziele werden dabei zum einen die Sicherheitskriterien des Konzeptes der Mehrseitigen Sicherheit verwendet. Die Notwendigkeit der Berücksichtigung weiterer Ziele wird 2.2 angesprochen. In [Re01] wird als Zielsystem ein Kriterienkatalog mit 79 Zielkriterien aus den Bereichen Sicherheit, Funktionalität und 
Wirtschaftlichkeit dargestellt. Risiko besteht folglich immer dann, wenn eine Abweichung von diesen Zielen festzustellen ist.

Da das mit einer Transaktion verbundene Risiko in Bezug auf die Anforderungen des Nutzers beurteilt werden muss, soll Risiko hier als eine subjektive, situationsabhängige Größe verstanden werden. Der Begriff Risiko wird hier demnach als die Möglichkeit verstanden, dass sich in einer bestimmten Transaktionssituation die Anforderungen eines Nutzers an die Sicherheit der Zahlungsdurchführung nicht erfüllen. In einem übertragenen Sinne entstehen auch Risiken, wenn sich die Anforderungen des Nutzers an weitere Eigenschaften elektronischer Zahlungssysteme wie die Funktionalität und die Wirtschaftlichkeit der Zahlungsdurchführung nicht erfüllen.

Risiken, die dem Nutzer bewusst sind und die entweder akzeptiert oder denen er durch Verwendung geeigneter Sicherheitsmaßnahmen entgegnet, werden hier als Restrisiko bezeichnet.

\subsection{Risikoanalyse: Bewertung elektronischer Zahlungssysteme}

Mit der Bewertung elektronischer Zahlungssysteme durch eine qualitative Risikoanalyse wird die Grundlage für die Einschätzung und Handhabung der mit der Verwendung elektronischer Zahlungssysteme verbundenen Risiken geschaffen und damit die Transparenz für den Nutzer erhöht. Dazu müssen aus der Sicht der Nutzer die Auswirkungen der Verwendung eines Zahlungssystems auf die Erfüllung von Sicherheits-, Funktionalitäts- und Wirtschaftlichkeitsanforderungen untersucht und bewertet werden.

Da bei der Auswahl von elektronischen Zahlungssystemen verschiedenste Aspekte aus den Bereichen Sicherheit, Funktionalität und Wirtschaftlichkeit einzubeziehen sind, die auch nicht-monetär erfassbare Größen beinhalten, wird auf das ScoringVerfahren als geeignete Vorgehensweise zur Durchführung der qualitativen Risikoanalyse zurückgegriffen. Das Scoring-Verfahren hilft dem Nutzer, rationale Entscheidungen zu treffen. Durch die transparente Vorgehensweise wird es auch Nicht-Experten möglich, diese Entscheidungen nachzuvollziehen.

In der Literatur findet man bereits ähnliche Ansätze zur Bewertung von Sicherheitseigenschaften unter Verwendung des Scoring-Verfahrens. So beschreiben etwa [RöKnNo00] ihr Vorgehen beim Quantifizieren der Sicherheit von E-Business-Anwendungen. Der dabei vorgeschlagene Ansatz beschränkt sich jedoch im Gegensatz zur hier vorgestellten Vorgehensweise lediglich auf die Bewertung der abstrakten Sicherheitsanforderungen Vertraulichkeit, Integrität, Verfügbarkeit und Verbindlichkeit. Zusätzliche Kriterien für die Nutzung von IT-Systemen wie die Funktionalität und die Wirtschaftlichkeit werden dabei nicht betrachtet.

Die Anpassung des Scoring-Verfahrens für die qualitative Risikoanalyse elektronischer Zahlungssysteme, die Festlegung der Untersuchungsreichweite, die Ergeb- 
nisse der Bewertung sowie die ausführliche Begründung der Bewertung sind in [Re01] dargestellt. Die Ergebnisse dieser Bewertung gehen als Zahlungssystemprofile in den Prozess der Risikohandhabung ein.

\subsection{Individuelle Risikohandhabung}

Für die Abwicklung einer Zahlungstransaktion muss der Nutzer eine Entscheidung zugunsten eines Zahlungssystems treffen, das sich in seinem Portfolio befindet. Aufgrund der Komplexität der Wirkungszusammenhänge und fehlender objektiver Informationen über die Auswirkungen seiner Zahlungssystemwahl läuft er Gefahr, dass seine Anforderungen verletzt werden. Obwohl die aktuell projektierten ECommerce-Systemarchitekturen die Verwaltung und Auswahl der dem Nutzer verfügbaren elektronischen Zahlungssysteme vereinfachen, wird das Problem der fehlenden Transparenz nicht behoben.

Diese Problematik kann durch eine Beratungs- und Entscheidungsunterstützungskomponente gelöst werden. Der Begriff „Virtual Internet Payment Assistant“ steht für diese Beratungs- und Entscheidungsunterstützungskomponente, die dem Nutzer als Ratgeber bei der Durchführung von Zahlungstransaktionen „virtuell“ zur Seite steht. Für die Auswahl elektronischer Zahlungssysteme bei Zahlungen im Internet existiert bislang noch kein Ansatz dieser Art. Im folgenden wird deshalb das Konzept eines virtuellen Assistenten zur Risikohandhabung skizziert, der dem Nutzer bereits vor der Durchführung von Zahlungstransaktionen beim Aufbau seines Zahlungssystemportfolios und in einer konkreten Zahlungssituation eine Einschätzung des mit der Wahl eines Zahlungssystems verbundenen transaktionsbezogenen Risikos und eine angemessene Reaktion zum Umgang mit diesen Risiken ermöglichen wird.

Wesentliches Element der Beratungs- und Entscheidungsunterstützungskomponente ist die Teilnahme des Nutzers an der Entscheidungsfindung, in dem er seine Anforderungen an die Zahlungstransaktion formulieren kann. Zur Vereinfachung der Anforderungsermittlung dienen hier Anforderungsprofile, deren Erstellung in 3 beschrieben wird.

\subsection{Der Virtual Internet Payment Assistant}

Der hier vorgestellte Virtual Internet Payment Assistant soll zwei Funktionen erfüllen: Die Beratung und die Entscheidungsunterstützung bei der Zahlungssystemwahl.

- Beratung: Der Beratungsdialog des Assistenten bietet dem Nutzer allgemeine, erklärende Informationen über die zur Auswahl stehenden Zahlungssysteme (etwa über den Betreiber des Zahlungssystems, den Zeitpunkt der Belastung, 
über eingesetzte Verschlüsselungsalgorithmen) und Erläuterungen zu der Bedeutung der Sicherheits-, Funktionalitäts- und Wirtschaftlichkeitskriterien.

- Entscheidungsunterstützung: Die Dialoge zur Entscheidungsunterstützung dienen dazu, einerseits die Nutzeranforderungen an die Sicherheit einer Transaktion zu ermitteln und andererseits basierend auf den durch die Risikoanalyse ermittelten Zahlungssystemprofilen elektronischer Zahlungssysteme und den Anforderungsprofilen der Nutzer die Wahl eines für die Transaktion geeigneten Zahlungssystems zu unterstützen. Die Entscheidungsunterstützung erfolgt (transaktions-) bzw. situationsbezogen.

Der Virtual Internet Payment Assistant erfüllt diese Funktionen dabei in zwei unterschiedlichen Anwendungsszenarien: Einerseits unterstützt er den Nutzer beim Aufbau seines Zahlungssystemportfolios vor der Durchführung von Zahlungstransaktionen (vgl. 2.4.1). Andererseits unterstützt er den Nutzer bei der Durchführung von Zahlungstransaktionen (vgl. 2.4.2). Passend dazu kann man Anforderungen unterscheiden in:

- Anforderungen, die Nutzer unabhängig von bzw. vor einer konkreten Zahlungstransaktion stellen können (,Situationsunabhängige“ Anforderungen für die Zahlungssystemwahl zum Zahlungsportfolioaufbau);

- Anforderungen, die Nutzer bezüglich der konkreten Zahlungstransaktion stellen können (,Situationsabhängige“ Anforderungen bei der Durchführung der Zahlungstransaktion) und

- Anforderungen, die Nutzer sowohl bei der Zahlungssystemwahl zum Zahlungsportfolioaufbau als auch bei der Durchführung der Zahlungstransaktion stellen können.

Eine Unterscheidung in situationsabhängige und situationsunabhängige Anforderungen erscheint u.a. deshalb sinnvoll, weil situationsabhängige Anforderungen zwar für die Wahl des Zahlungssystems in einer konkreten Zahlungssituation wichtig sind, generell aber auch für die situationsunabhängige Entscheidung zum Einsatz eines Zahlungssystems von Bedeutung sind. Rein situationsunabhängige Anforderungen greifen dagegen lediglich bei der grundsätzlichen Entscheidung über den Einsatz eines elektronischen Zahlungssystems bzw. über die Aufnahme eines elektronischen Zahlungssystems in das Portfolio des Nutzers.

\subsubsection{Aufbau des Zahlungssystemportfolios}

Beim Aufbau des Zahlungssystemportfolios steht dem Nutzer eine große Zahl an elektronischen Zahlungssystemen zur Verfügung, die in ihren Sicherheits-, Funktionalitäts- und Wirtschaftlichkeitseigenschaften in der Regel stark variieren. Die in Form der Zahlungssystemprofile vorliegenden Ergebnisse der Risikoanalyse bestätigen diese Aussage (vgl. [Re01]). 
Die Nutzer stellen in Bezug auf unterschiedliche Transaktionssituationen durchaus auch unterschiedliche Anforderungen an die Eigenschaften der Zahlungssysteme [Ki00]. Da zwischen der Entscheidung zugunsten des Einsatzes eines Zahlungssystems und einer wirklichen Einsetzbarkeit in der Regel mehrere Tage vergehen, muss sich der Nutzer rechtzeitig vor der beabsichtigten Nutzung des Zahlungssystems Gedanken über die Auswahl der für ihn geeigneten Zahlungssysteme machen.

Der Virtual Internet Payment Assistant unterstützt den Nutzer in diesem Anwendungsszenario, lange vor der eigentlichen Durchführung von Zahlungstransaktionen aus der Vielzahl von Zahlungssystemalternativen die für ihn geeigneten Zahlungssysteme auszuwählen.

\subsubsection{Durchführung von Zahlungstransaktionen}

Das zweite Anwendungsszenario liegt in der Entscheidungsunterstützung zum Zeitpunkt der Durchführung von Zahlungstransaktionen. Nachdem der Nutzer die Ware auf den Webseiten des Händlers ausgesucht hat, muss er sich vor der endgültigen Bestätigung der Bestellung für ein Bezahlverfahren entscheiden. Neben traditionellen Verfahren wie der Übermittlung eines Schecks als Vorkasse oder der Banküberweisung nach Rechnungsstellung stehen ihm mittlerweile eine Reihe elektronischer Zahlungssysteme für die Zahlung zur Verfügung. Aus der Schnittmenge der vom Händler zur Zahlung angebotenen Zahlungssysteme und der Zahlungssysteme im Zahlungssystemportfolio des Nutzers muss der Nutzer ein Zahlungssystem auswählen, das seinen Anforderungen am Nächsten kommt.

Der Virtual Internet Payment Assistant unterstützt den Nutzer dabei, aus dieser Schnittmenge von Zahlungssystemalternativen das für ihn am besten geeignete Zahlungssystem auszuwählen.

\section{Anforderungsprofile als Grundlage für die individuelle Risikohandhabung}

Vor der Durchführung einer Zahlungstransaktion werden die Anforderungen des Nutzers an die Eigenschaften ,seiner“ elektronischen Zahlungssysteme zur Zahlungsabwicklung festgelegt.

Würde der Nutzer mit der vollen Bandbreite der im Kriterienkatalog dargestellten Anforderungen konfrontiert, wäre er als Laie überfordert. Der Konfigurationsprozess würde vom Nutzer Expertenwissen voraussetzen und zu viel Zeit in Anspruch nehmen. Aus diesem Grund müssen für den Nutzer verständliche, von technischen Details abstrahierte Sicherheits-, Funktionalitäts- und Wirtschaftlichkeitsziele gefunden werden, mit denen er seine Anforderungen beim Aufbau seines Zah- 
lungssystemportfolios und bei der Durchführung der Zahlungstransaktion beschreiben kann. Die Abstraktionen dieser ursprünglich eher technischen Ziele orientieren sich dabei an den (subjektiven) Zielen der Nutzer.

Zur Erfassung dieser Anforderungen sollten die Eigenschaften der die elektronischen Zahlungssysteme beschreibenden Zielkriterien einfach und verständlich dargestellt sein. In 3.1 wird deshalb ein Modell vorgestellt, das eine Abstrahierung und Gliederung der Anforderungen in Ebenen von Anforderungsprofilen vorschlägt. Auf die Umsetzung der gewählten Vorgehensweise zur Erstellung der Anforderungsprofile durch Anwendung des Scoring-Verfahrens wird anschießend in 3.2 eingegangen.

\subsection{Ebenenmodell für Anforderungsprofile}

Um dem Nutzer einerseits die Konfiguration seiner Anforderungen zu vereinfachen und andererseits eine möglichst detaillierte und auf den jeweiligen Transaktionstyp bzw. auf die jeweilige (Transaktions-)Situation angepasste Feineinstellung seiner Anforderungen zu ermöglichen, wird die Konfiguration der Nutzeranforderungen in drei Ebenen eingeteilt (vgl. Abbildung 1).

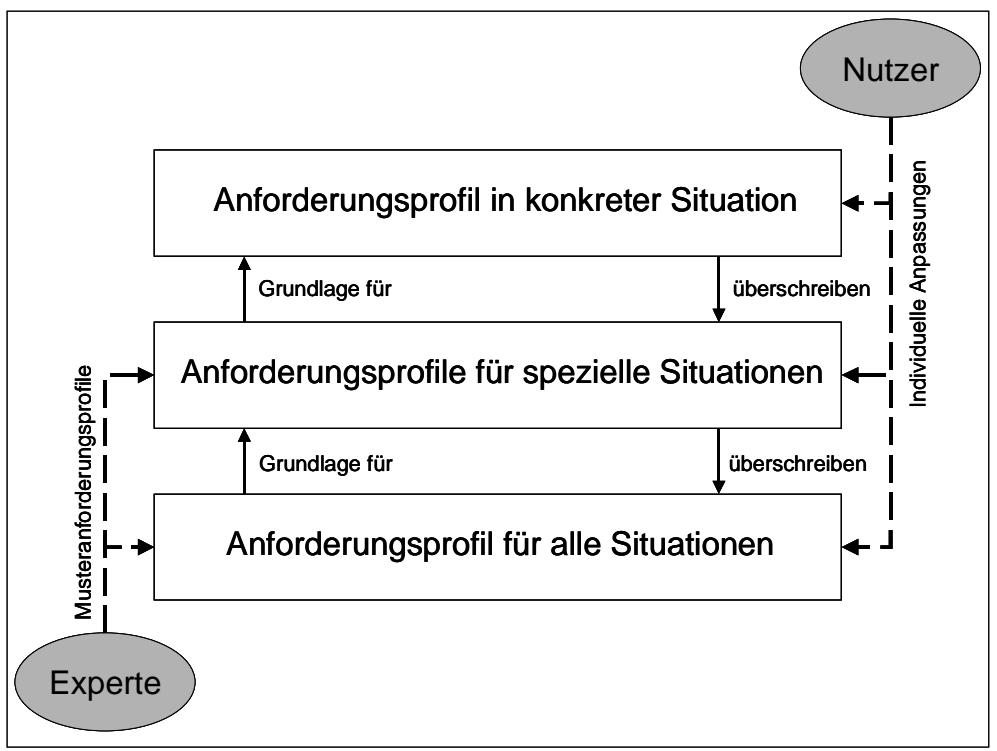

Abbildung 1: Ebenenmodell für Anforderungsprofile

Anforderungsprofile auf einer höheren Ebene bauen auf Anforderungsprofilen der darunter liegenden Ebenen auf. Geforderte Eigenschaften im Profil für alle Situationen gelten auch in Anforderungsprofilen für spezielle Situationen und in der konkreten Situation, solange diese Anforderung in einem der Anforderungsprofile 
auf einer höheren Ebene keine andere Einstellung aufweist. In diesem Falle würde die Einstellung auf der unteren Ebene ,überschrieben“.

Den Ebenen entsprechend werden drei Typen von Anforderungsprofilen geführt:

- Ein Anforderungsprofil für alle Situationen;

- Anforderungsprofile für bestimmte Situationen und

- ein Anforderungsprofil für die konkrete Situation.

\subsubsection{Anforderungsprofil für alle Situationen}

Auf der ersten Ebene sind Anforderungen konfiguriert, die grundsätzlich als Vorannahme in jeder Situation gelten sollen.

Der Nutzer kann einerseits das von Experten vorkonfigurierte Musteranforderungsprofil für alle Situationen nutzen, andererseits jedoch auch selbst eigene Einstellungen an diesem Anforderungsprofil vornehmen.

\subsubsection{Anforderungsprofil für bestimmte Situationen}

Auf der zweiten Ebene sind Anforderungen konfiguriert in Bezug auf bestimmte Typen von Transaktionen und Situationen, etwa für die Geschäftsbeziehung mit bestimmten Partnern. Dementsprechend werden hier beispielhaft Anforderungsprofile für die folgenden Transaktionstypen bzw. (Transaktions-)Situationen angenommen:

- Der Nutzer fordert eine minimale Sichtbarkeit der Transaktion gegenüber anderen Teilnehmern im Internet (Anforderungsprofil mit Schwerpunkt auf Sicherheitskriterien).

- Der Nutzer fordert eine maximale Bedienbarkeit des Zahlungssystems bei der Durchführung der Zahlungstransaktion (Anforderungsprofil mit Schwerpunkt auf Funktionalitätskriterien).

- Der Nutzer fordert eine wirtschaftliche und kostengünstige Durchführung der Zahlungstransaktion (Anforderungsprofil mit Schwerpunkt auf Wirtschaftlichkeitskriterien).

Erfahrungen aus Forschungsprojekten mit ähnlichem Lösungsansatz (vgl. [DaPoRe99]) haben gezeigt, dass dem Nutzer die Auswahl unter drei bis vier verschiedenen (Muster-)Anforderungsprofilen zur Handhabung der meisten Situationen ausreicht. Eine größere Anzahl von für bestimmte Situationen vordefinierten Profilen erhöht die Komplexität für den Nutzer.

Der Nutzer kann einerseits die von Experten vorkonfigurierten Musteranforderungsprofile für besondere Situationen nutzen, andererseits jedoch auch selbst eigene Anforderungsprofile für zusätzliche Situationen definieren. 


\subsubsection{Anforderungsprofil für konkrete Situation}

Der Nutzer hat bei diesem Ansatz zwar die Möglichkeit, unter von Experten vorkonfigurierten Anforderungsprofilen auszuwählen. Er kann jedoch bei der Durchführung der Transaktion zusätzlich jederzeit auf einzelne Einstellungen einwirken bzw. entgegen der Empfehlung des Virtual Internet Payment Assistants eine andere Auswahl treffen. Der Virtual Internet Payment Assistant informiert den Nutzer über mögliche Konsequenzen seiner Änderung, auch im Hinblick auf Folgen der Kombination verschiedener Anforderungen.

Dass diese Möglichkeit sinnvoll erscheint, haben bereits die in der Simulationsstudie des Kollegs „Mehrseitige Sicherheit in der Kommunikationstechnik“ der Gottlieb Daimler- und Karl Benz-Stiftung gemachten Erfahrungen mit situationsbedingten Einstellungen der (telefonischen) Erreichbarkeit gezeigt. Diese Erfahrungen weisen darauf hin, dass Nutzer zwar in der Regel die in einem Mustersituationsprofil vorkonfigurierte Einstellung eines Experten nutzen, dass sie jedoch trotzdem gerne die Möglichkeit haben, zum Zeitpunkt eines Telefonates individuell reagieren zu können [DaPoRe99].

Auf der dritten Ebene konfiguriert bzw. ändert der Nutzer deshalb seine Anforderungen in der konkreten Transaktionssituation bei der Durchführung einer Zahlung. Die Voreinstellungen, die auf der ersten und zweiten Ebene konfiguriert sind, kann der Nutzer in einer konkreten Transaktionssituation (auf der dritten Ebene) überschreiben.

Der Virtual Internet Payment Assistant „,merkt“ sich diese Änderungen des Nutzers an Anforderungsprofilen und schlägt sie dem Nutzer beim Wiederholen ähnlicher Transaktionssituationen, etwa beim wiederholten Besuch eines bestimmten Händlers, zur Unterstützung des Nutzers als Änderung des Anforderungsprofils vor (vgl. Ausführungen zur Nutzer-Partizipation in [Re94]).

\subsubsection{Musteranforderungsprofile}

Der Virtual Internet Payment Assistant bietet zur Vereinfachung von Experten erstellte Musteranforderungsprofile mit plausiblen Voreinstellungen $\mathrm{zu}$ den Sicherheits-, Funktionalitäts- und Wirtschaftlichkeitsanforderungen für die ersten beiden Ebenen an.

Der Nutzer sollte dabei grundsätzlich die Möglichkeit haben, diese Musteranforderungsprofile der ersten beiden Ebenen abzuändern und dementsprechend die Voreinstellungen für die Anforderungen in bestimmten Situationen seinen eigenen Bedürfnissen anzupassen [DiFiAbBe98]. Der Virtual Internet Payment Assistant unterstützt den Nutzer dabei, Wechselwirkungen zwischen der Erfüllung einzelner Anforderungen zu berücksichtigen. 


\subsection{Realisierung der Anforderungsprofile mit dem Scoring- Verfahren}

Zur Realisierung der Anforderungsprofile werden die in 2.1 angesprochenen Bewertungskriterien herangezogen. Nach einer Unterteilung dieser Kriterien in Muss- und Kannkriterien wird die Vorgehensweise bei der Gewichtung der Kannkriterien nach dem Scoring-Verfahren beschrieben.

\subsubsection{Unterscheidung von Muss- und Kannkriterien}

Der erste Schritt zur Erzeugung der Anforderungsprofile ist die Unterteilung der Bewertungskriterien in Muss- und Kannkriterien (vgl. Abbildung 2).

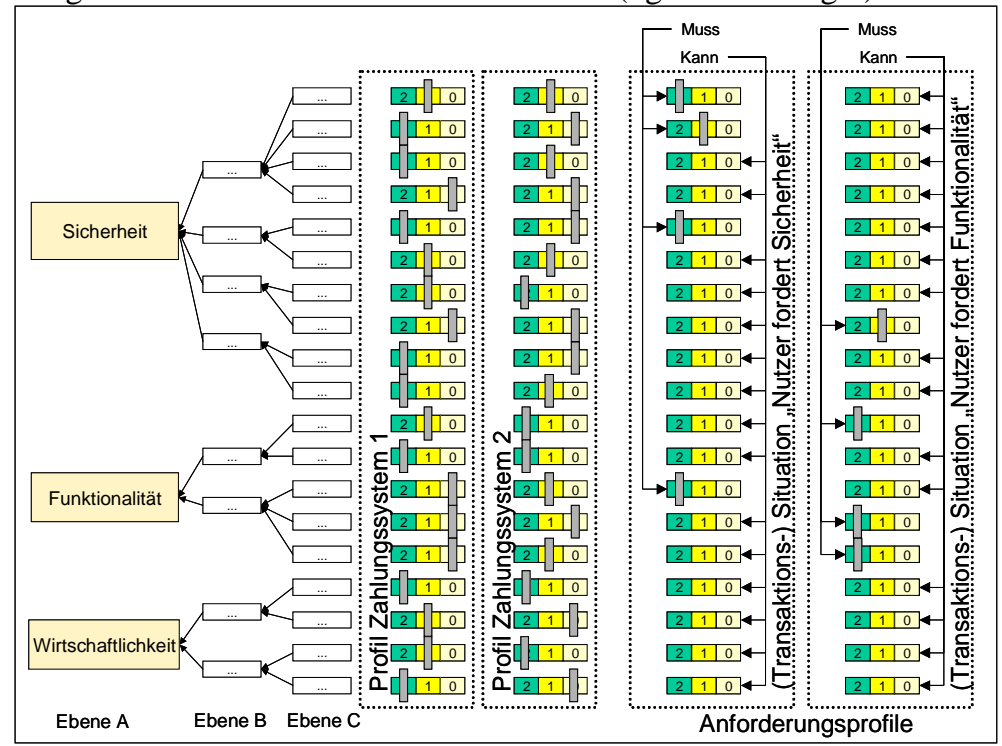

Abbildung 2: Darstellung der Anforderungsprofile mit Muss- und Kannkriterien

Als Musskriterien bezeichnet man Kriterien, deren Nichterfüllung bzw. Nichtvorhandensein zur Aussonderung der Alternative führt. Dies gilt bereits für die Nichterfüllung eines einzigen Musskriteriums, selbst wenn alle anderen erfüllt sind. Ein Musskriterium gilt als erfüllt, wenn die geforderte Ausprägung des Kriteriums im Anforderungsprofil mit der Ausprägung dieses Kriteriums im Zahlungssystemprofil übereinstimmt bzw. wenn die geforderte Ausprägung des Kriteriums im Anforderungsprofil niedriger ist wie die Ausprägung dieses Kriteriums im Zahlungssystemprofil. Ist beispielsweise die volle Gewährleistung ,Vertraulichkeit der Kundeninformation“ gefordert und das Zahlungssystemprofil eines der auf dem Rechner des Nutzers verfügbaren elektronischen Zahlungssysteme zeigt für dieses Kriterium nur eine eingeschränkte Gewährleistung an, wird die Alternative 
ausgesondert. Ein Bewertungskriterium darf gerne übererfüllt sein, der umgekehrte Fall führt zur Aussonderung des Zahlungssystems.

Kannkriterien sind alle diejenigen Kriterien, deren Vorhandensein bzw. deren Erfüllung für den Nutzer als Entscheidungsträger bedeutsam sind, deren Nichterfüllung bzw. Nichtvorhandensein jedoch nicht zur Aussonderung der Alternative führt. Den einzelnen Kannkriterien bzw. Anforderungen kann in einem weiteren Schritt eine unterschiedliche Bedeutung beigemessen werden, indem sie unterschiedlich stark gewichtet werden.

\subsubsection{Vorgehensweise bei der Gewichtung}

Die relative Gewichtung der Kannkriterien zueinander richtet sich nach den individuellen Anforderungen eines Nutzers an die durchzuführende Transaktion (vgl. Abbildung 3). Die vertikalen Balken in den Anforderungsprofilen von Abbildung 3 sollen durch die Schattierung eine unterschiedliche Gewichtung einzelner Anforderungen symbolisieren.

Die Ausführungen in 3.1 zeigen, dass es für den Nutzer einfacher wäre, wenn er statt der individuellen Konfiguration aller Kriterien auf der untersten Ebene lediglich die gewünschte Erfüllung von 3 bis 5 abstrahierten Eigenschaften elektronischer Zahlungssysteme derart bestimmen müsste, dass sie seinen Präferenzen am nächsten kommen. Da diese Gewichtung „situationsspezifisch“ entstehen wird, können sich Unterschiede in der Gewichtung, beispielsweise in der Art des Gutes (physisch oder digital), nach der Höhe des Preises, der Höhe der Transaktionskosten, der Vertrauenswürdigkeit des Händlers, der Dauer der Lieferung oder der Haftung im Verlustfalle ausdrücken.

Der Einfachheit halber werden deshalb als abstrahierte Eigenschaften die drei Kriteriengruppen Sicherheits-, Funktionalitäts- und Wirtschaftlichkeitsanforderungen an die Durchführung einer Zahlungstransaktion angenommen.

Da man beim durchschnittlichen Nutzer im Allgemeinen von einer fehlenden sachlichen Kompetenz ausgehen muss, sollte die weitergehende Gewichtung der übrigen Kriterien vorab von Experten erstellt (vgl. die Ausführungen in 3.1 und [Za76]) und dem Nutzer plausible, situationsspezifische Anforderungsprofile vorgeschlagen werden. Der Nutzer wird mit Unterstützung des Virtual Internet Payment Assistenten demnach in der Regel lediglich die Gewichte dieser „einfachen“, abstrahierten Eigenschaften variieren und im Übrigen ein jeweils zur Transaktionssituation passendes (Muster-)Anforderungsprofil auswählen. 


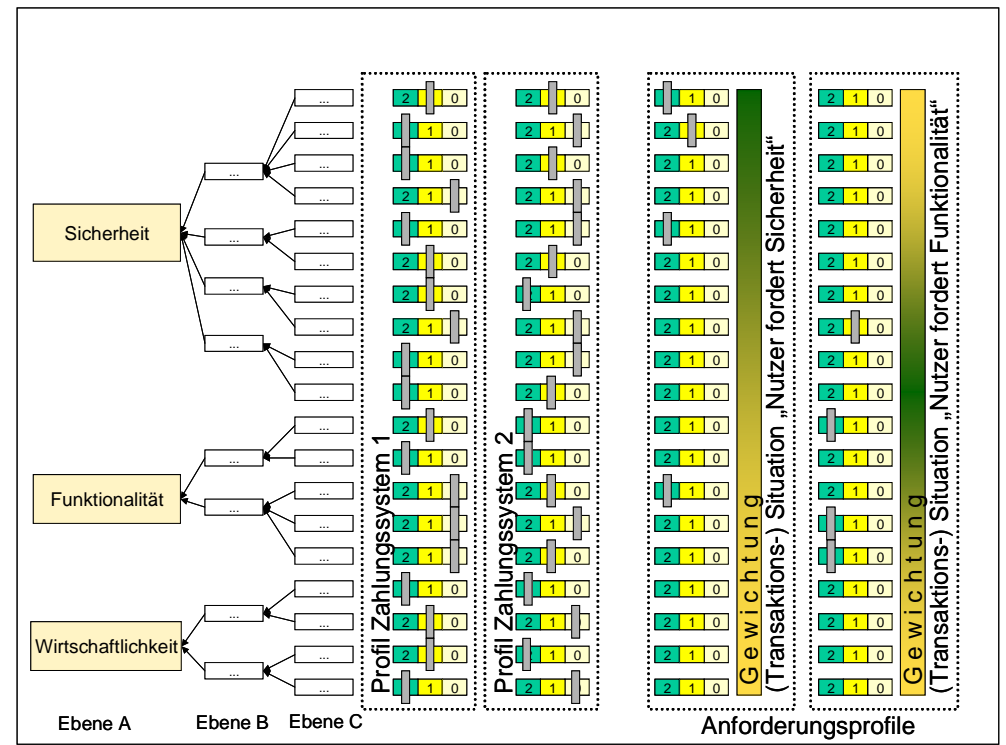

Abbildung 3: Symbolische Darstellung der Gewichtung in Anforderungsprofilen

Erkenntnisse über das Zahlungssystemwahlverhalten von Nutzern in bestimmten Situationen, die sich zur Erstellung dieser Musteranforderungsprofile verwenden ließen, liegen u.a. in [Ki00] vor.

\subsubsection{Berücksichtigung von Wechselwirkungen}

Bei der Erstellung von Anforderungsprofilen lassen sich aufgrund der Wechselwirkungen zwischen einzelnen Kriterien bestimmte Konstellationen gewünschter Eigenschaften von vorneherein ausschließen. Beispielsweise macht es wenig Sinn, in dem Anforderungsprofil einer fiktiven Situation „Der Nutzer fordert eine minimale Sichtbarkeit der Transaktion gegenüber anderen Teilnehmern im Internet“" sowohl Anonymität als auch Nachweisbarkeit der Transaktion zu fordern, da keines der gegenwärtig verfügbaren elektronischen Zahlungssysteme beide Anforderungen gleichzeitig erfüllt.

\subsubsection{Sensitivitätsanalysen über die Gewichte}

In Anwendungsfällen des Scoring-Verfahrens wie diesem, in denen auf der Basis exakter Gewichte bestimmte Werte der alternativen elektronischen Zahlungssysteme punktgenau abgeleitet werden, könnte man in weiteren Schritten überlegen, ob sich nicht durch geringe Änderungen der (Gewichts-)Parameter die Empfehlung zur Zahlungssystemwahl ändern würde. 
Hat der Nutzer beispielsweise Gewichte ermittelt, die zur Auswahl der Kreditkarte führen, ist es nun noch von Interesse, ob die Entscheidung für die Kreditkarte relativ stabil ist oder schon bei geringfügigen Gewichtsverschiebungen umkippt, etwa zugunsten einer Zahlung mit dem Zahlungssystem eCash. Das elektronische Zahlungssystem eCash ist in dem Attribut „Vertraulichkeit der Kundeninformationen“ der Kreditkarte überlegen. Ein höheres Gewicht für die Anforderung „Vertraulichkeit der Kundeninformationen“ könnte also dazu führen, dass das Zahlungssystem eCash einen höheren Nutzwert erhält als die Kreditkarte.

Wegen der Normierung der Gewichte müssen Annahmen getroffen werden, wie sich die anderen Gewichte ändern, wenn das Gewicht eines Attributs variiert. Demnach ist es nicht zulässig, die Auswirkungen einer isolierten Erhöhung des Gewichts für die Sicherheit zu testen. Eine mögliche Annahme ist, dass sich bei der Erhöhung des Gewichts der Sicherheitsanforderungen die Gewichte der anderen Attribute so verringern, dass sie stets im selben Verhältnis zueinander stehen wie in der Ausgangssituation.

\section{Ausblick}

Nachdem in 3 beschrieben worden ist, wie die Anforderungsprofile für Nutzer zur Vorbereitung der Risikohandhabung erstellt werden, sind die Grundlagen für den eigentlichen Vorgang der Risikohandhabung beim Aufbau des Zahlungssystemportfolios bzw. bei der Durchführung der Transaktion geschaffen. Dabei werden schließlich die Anforderungsprofile mit den durch die Zahlungssysteme erreichbaren ,technischen Sicherheitslevel“ bzw. mit den Zahlungssystemprofilen abgeglichen und dem Nutzer das für die Transaktion am besten geeignete Zahlungssystem vorgeschlagen. Damit wurden die Voraussetzungen für die Realisierung eines Virtual Internet Payment Assistants geschaffen, der den Nutzern das Vertrauen in die korrekte Funktionsweise der elektronischen Zahlungssysteme und damit die Kontrolle über ihre Privatsphäre zurückgeben wird.

\section{Literatur}

[DaPoRe99] Damker, H.; Pordesch, U.; Reichenbach, M.: Personal Reachability and Security Management - Negotiation of Multilateral Security. In: [MüRa99], S. 95-111.

[DiFiAbBe98] Dix A.J.; Finlay J.E.; Abowd G.D.; Beale R. : Human-Computer Interaction. Second Edition. Prentice Hall Europe, 1998.

[EgEn00] Eggs, H.; Englert, J.: Electronic Commerce Enquête II - Business-to-Business Electronic Commerce, Empirische Studie zum Business-to-Business Electronic Com- 
merce im deutschsprachigen Raum. Executive Research Report, Konradin-Verlag, Stuttgart 2000.

[EgMü01] Müller, G.;Eggs, H.: Sicherheit und Vertrauen: Mehrwert im E-Commerce. In: [MüRe01], S. 27-43.

[Fo00] Fox, S.: Trust and privacy online - Why Americans want to rewrite the rules. The Internet Life Report, The Pew Internet \& American Life Project, August 2000.

[JeMa00] Jendricke, U.; Markotten, D. G. t.: Usability meets Security - The Identity-Manager as your Personal Security Assistant for the Internet. In: Proceedings of the ACSAC 2000, New Orleans, USA, 11.-15. Dezember, 2000.

[Ki00] Kiefer, T.: Trust Mediation durch Banken im Electronic Commerce. Gabler Verlag, 2001 .

[MüRa99] Müller, G.; Rannenberg, K. (Hrsg.): Multilateral Security for Global Communication - Technology, Application, Business. Vol. 3, Addison-Wesley-Longman, 1998, Bonn; Reading, Massachusetts, Juli 1999.

[MüRe01] Müller, G.; Reichenbach M. (Hrsg.): Sicherheitskonzepte für das Internet, Springer Xpert.press, Mai 2001.

[Re01] Reichenbach, M.: Individuelle Risikohandhabung elektronischer Zah-lungssysteme. Gabler-Verlag/Deutscher Universitätsverlag, Gabler edition Wissenschaft, Herausgegeben von Picot, A.; Reichwald, R., Reihe "Markt- und Unternehmensentwicklung", Wiesbaden 2001.

[Re94] Reisin, F.-M.: Software-Ergonomie braucht Partizipation. In: E. Eberleh, H. Oberquelle, R. Oppermann (Hrsg.): Einführung in die Software-Ergonomie, 2. Auflage, Berlin - New York, Walter de Gruyter, 1994, S. 299-333.

[RöKnNo00] Röhrig, S.; Knorr, K.; Noser H.: Sicherheit von E-Business-Anwendungen Struktur und Quantifizierung. In: Wirtschaftsinformatik, Verlag Vieweg, Dezember 2000 .

[Za76] Zangemeister, C.: Nutzwertanalyse in der Systemtechnik - Eine Methodik zur multidimensionalen Bewertung und Auswahl von Projektalternati-ven. 4. Aufl., München, 1976. 